\title{
Hábitos higiénicos orales en escolares de Pachacútec, Ventanilla, Callao
}

Oral hygiene habits among students from the Pachacútec, Ventanilla, Callao

\begin{abstract}
Resumen
Se realizó una encuesta cuyo objetivo fue evaluar los hábitos higiénicos orales en escolares de la Institución Educativa (IE) N ${ }^{\circ} .5130$ de Pachacútec Ventanilla, Callao. Se tomó a la totalidad de la población (225 nińos) de las edades índice 6, 12 y 15 años. Los resultados mostraron que el cepillado diario más de una vez al día fue realizado por el $46,7 \%$ de los nińos y una vez al día por el $21,3 \%$. Un 10,7 \% no se cepilla nunca y un $20,0 \%$ lo hace de vez en cuando. Un 34,7 \% ha ido al dentista en los últimos seis meses. Un 9,3\% lo hizo entre los seis meses y el ańo, y $26,7 \%$ no ha ido nunca al dentista. El 18,7 \% ha hecho enjuagues de flúor durante dos o más ańos, $9,3 \%$ ha hecho enjuagues solo durante un año y el 72,0 \% nunca ha hecho enjuagues con flúor en la IE. Se concluye que los hábitos higiénicos orales de los escolares del I.E.5130 de Pachacútec de edades índice 6, 12 y 15 indican que hay la necesidad de realizar un programa de enjuagatorios sostenido, y educación para la salud para mejorar los conocimientos de los escolares acerca de la prevención de la caries y para mejorar los hábitos de higiene oral logrando incremento de la frecuencia de cepillados diarios.

Palabras clave: Epidemiología dental, salud oral, hábitos de salud oral, escolares, cepillado dental, enjuagues de flúor.
\end{abstract}

\begin{abstract}
A survey was performed which objective was to evaluate the oral hygiene habits among students from the Educational Institution (IE) No. 5130 at Pachacútec, Ventanilla, El Callao. The entire population was taken (225 children) who were 6, 12 and 15 years old. The results showed that $21.3 \%$ and $46.7 \%$ of the children brush their teeth once a day and more than once a day, respectively; $10.7 \%$ of them never brushes their teeth and $20 \%$ practices it sometimes. $34.7 \%$ of them has visited the dentist in the last six months, while $9.3 \%$ did it between the last six months to a year, and $26.7 \%$ has never gone to the dentist. $18.7 \%$ has done fluoride mouthwashing during two or more years, $9.3 \%$ has done fluoride mouthwashing just during one year, and $72.0 \%$ has never done fluoride mouthwashing at the Educational Institution. It is concluded that the oral hygiene habits of the 6,12 and 15-year-old students from the Educational Institution No. 5130 at Pachacútec show that there is a need to develop a sustained mouthwashing program and health education to improve oral hygiene habits increasing the tooth brushing frequency.

Key words: dental epidemiology, oral health, oral health habits, students, tooth brushing, fluoride
\end{abstract} mouthwashing.

\section{Introducción}

La adopción de una política de prevención de las enfermedades y de promoción de la salud, tanto del individuo como del ambiente en que vive, ayudaría a mejorar la calidad y las expectativas de vida de la población en mayor medida que cualquier otra política. No hay que olvidar que existen no solo individuos sanos e individuos enfermos sino también poblaciones sanas y poblaciones enfermas. ${ }^{1}$

La nueva Salud Pública intenta dar respuesta a la crisis de los sistemas de asistencia sanitaria reconociendo la importancia del ambiente, tanto físico como social, en la salud. Considera los estilos de vida en un contexto de conductas saludables (creación de entorno saludable) en un marco social donde inciden las políticas sanitarias, trata de conseguir que las opciones más saludables sean las más fáciles de escoger y de llevar a la práctica. Se preocupa del desarrollo de las habilidades personales que conducen a cambio de comportamientos relacionados con la salud, la reorientación de los servicios sanitarios y de salud pública; trata de construir cultura sanitaria. ${ }^{2}$

Es decir que en la actualidad, la salud pública tiene un enfoque más global acerca de la salud y de las enfermedades bucodentales, y tras el fracaso de la odontología paliativa en el control de la enfermedad, surgió una nueva perspectiva para enfrentarse a los problemas de salud bucodental. Esta nueva perspectiva se fundamenta en la utilización de medidas preventivas aplicadas sobre

\section{Artículo Original}

\section{Marco Tulio Madrid Chumacero ${ }^{1}$, Antonia Castro Rodríguez ${ }^{2}$, Juana Echeandía Arellano ${ }^{3}$, Sylvia Chein Villacampa ${ }^{1}$, Jorge Villavicencio Gastelú ${ }^{4}$, Adelmo Cuentas Robles ${ }^{4}$, Ricardo Vásquez Olivares ${ }^{1}$}

\footnotetext{
${ }^{1}$ Profesor Asociado del Departamento Académico Estomatología Preventiva y Social FOUNMSM

${ }^{2}$ Profesora Principal del Departamento Académico Estomatología Preventiva y Social FOUNMSM

3 Profesora Principal de la FM-UNMSM

${ }^{4}$ Profesor Asociado del Departamento Académico Médico Quirúrgico FO- UNMSM
}

Correspondencia:

CD Marco Tulio Madrid Chumacero

Facultad de Odontología

Universidad Nacional Mayor de San Marcos

E-mail: mmadridc@unmsm.edu.pe

Teléfono: 3323865

Dirección: Jr. Restauración 373. Dpto. 102.

Lima 5. Perú

Fecha de recepción: 20-08-10

Fecha de aprobación: 08-11-10

grandes grupos de población. La mayoría de estas medidas como la utilización de Flúor, mayor higiene oral y mejoramiento de los niveles de conciencia odontológica están relacionadas con cambios de los hábitos de comportamiento de la población. ${ }^{3,4}$

Las medidas preventivas aplicadas a nivel individual requieren la colaboración de cada individuo de la colectividad para llevarse a cabo: vacunaciones, uso del cinturón de seguridad, cepillado dental adecuado, utilización de hilo dental, dieta equilibrada, etc. De allí nace la estrategia de alto riesgo y la estrategia poblacional.

La estrategia de alto riesgo, a pesar de sus limitaciones (conceptuales y de índole practica) ejerce un mayor atractivo sobre una profesión de marcado carác- 
ter individualista como es la odontología. Sin embargo, al ser la mayoría de las enfermedades orales de carácter multifactorial, solo con la aplicación de un enfoque poblacional de promoción de la salud oral se conseguirá una disminución de su incidencia. ${ }^{5}$

La promoción de la salud, según la carta de Ottawa, es el proceso de capacitación del conjunto de individuos para que ejerzan un mayor control sobre su propia salud y puedan así mejorarla. ${ }^{6}$

La prevención y la promoción persiguen como meta la salud, pero la prevención lo hace situando su punto de referencia en la enfermedad. El objetivo de la prevención es evitar la enfermedad, mientras que el de la promoción es maximizar los niveles de salud.

La carta de Ottawa, elaborada en 1986, propuso cinco mecanismos para la promoción de la salud: 1 . Desarrollo de habilidades personales, 2. Reorientación de los servicios de salud, 3. Promoción de políticas saludables, 4. Fortalecimiento de la acción comunitaria, 5 . Creación de ambientes favorables.

La mayoría de las enfermedades orales, como la caries dental, las enfermedades periodontales, el cáncer oral, las alteraciones del flujo salival y los problemas de articulación temporo mandibular (ATM) tienen una relación directa con el consumo de azúcar, una higiene oral deficiente, el tabaco, el alcohol o el estrés. Difícilmente se pueden controlar estos problemas sin un enfoque global de promoción de la salud a través de la promoción de la salud oral. ${ }^{7}$

Frente a un modelo en el que se enfatizan las actuaciones preventivas sobre el individuo asumiendo que este es el único responsable de su estilo de vida y, por ello, de sus posibles conductas de riesgo, el modelo de promoción de la salud reconoce la necesidad de cambiar las condiciones en las que los individuos viven en un sentido más saludable.

El individuo es responsable de sus conductas de vida. Pero la sociedad es responsable de las condiciones de vida. Para conseguir la salud son necesarias determinadas condiciones de vida. Para conseguir la salud son necesarias determinadas condiciones sociales con el fin de que los individuos puedan de manera consciente mejorar su salud ${ }^{8}$.y para determinar las circunstancias que relacionan estilos de vida y salud bucodental se ha realizado esta encuesta sobre hábitos, coincidiendo con la realización de estudio epidemiológico de Diagnóstico de salud oral y necesidades de tratamiento y con motivo de la realización de programa de salud, dirigida a la población infantil de la Institución Educativa (IE) 5130 de Pachacútec,

Para Brathall ${ }^{9}$ el uso diario de pasta fluorada varias veces al día, es el más importante factor causante del descenso de la caries en los países desarrollados, lo que le confiere al cepillado regular el principal objetivo de introducir el flúor en el medio oral a través de la pasta dentífrica.

El presente trabajo muestra los hábitos de higiene oral y los conocimientos sobre prevención, flúor, y cariogenicidad de los alimentos que presentan los escolares de 6, 12 y 15 años de la IE $\mathrm{N}^{\circ}$ 5130 de Pachacútec, Ventanilla, Callao.

\section{Material y Método}

Se realizó el presente estudio transversal descriptivo aplicando un cuestionario después de examinar a los escolares de la IE. 5130; donde se logró el consentimiento de profesores, padres de familia y alumnos. Se escogieron las edades índices de 6, 12 y 15 ańos de acuerdo con las encuestas de salud bucodental: métodos básicos de la Organización Mundial de la Salud, $1997 .^{10}$

Este estudio sobre hábitos de higiene y conocimientos acerca de la prevención de caries dental se realizó de forma simultánea al estudio epidemiológico de salud buco dental realizado en escolares de la IE.5130 realizado por el mismo equipo de investigación

Se tomó a la totalidad de la población 225 de las edades índice mencionada. La encuesta se realizó una vez terminada la exploración oral, para lo cual se le proporcionaba el cuestionario de preguntas tipo test con múltiples respuestas que el propio alumno debía contesVentanilla, Callao.

tar de forma anónima. El trabajo en la IE demandó todo el mes de Noviembre del 2007.

Las preguntas abarcaban hábitos de higiene, asistencia al dentista, conocimientos sobre la utilidad del flúor, grado de cariogenicidad de ciertos alimentos, frecuencia de cepillado e ingesta de alimentos ricos en hidratos de carbono.

Se registraron las siguientes variables: Utilización de servicios odontológicos, Realización de enjuagues de flúor en el colegio, Conocimiento de la acción del flúor, Tiempo transcurrido desde la última visita al dentista, Motivo de la última visita al dentista, Sensación de ir al dentista, Número de cepillados, Conocimiento sobre si es beneficioso ingerir alimentos entre comidas, Ingestión de alimentos azucarados entre comidas, Conocimiento del grado de cariogenicidad de los distintos grupos de alimentos: carne y pescado, chocolate, frutas frescas, verduras y ensaladas, bebidas refrescantes, huevos y leche, helados, frutos secos, galletas y pasteles, golosinas y caramelos.

\section{Análisis estadístico}

Los datos obtenidos fueron almacenados en una base del programa EXCEL de Microsoft ${ }^{\oplus}$ y analizados mediante el programa estadístico SPSS $10.0^{\circ}$, realizándose una estadística descriptiva de las variables categóricas con proporciones.

\section{Resultados}

El $32 \%$ de los encuestados de 12 ańos y el $24 \%$ de los de 15 años han ido al dentista en los últimos seis meses. Un 8 $\%$ y un $12 \%$ lo hicieron entre los seis meses y el año.

El motivo de consulta más frecuente fue caries, dolor (24\%) y otros motivos

Tabla 1. Utilización de los servicios odontológicos.

\begin{tabular}{|c|c|c|c|c|c|c|c|c|c|}
\hline \multirow{2}{*}{\multicolumn{2}{|c|}{ Utilización de los servicios odontológicos }} & \multicolumn{2}{|c|}{ Total } & \multicolumn{2}{|c|}{6 años } & \multicolumn{2}{|c|}{12 años } & \multicolumn{2}{|c|}{15 años } \\
\hline & & $\mathrm{n}$ & $\%$ & $\mathrm{n}$ & $\%$ & $\mathrm{n}$ & $\%$ & $\mathrm{n}$ & $\%$ \\
\hline \multirow{4}{*}{$\begin{array}{l}\text { Tiempo transcurrido } \\
\text { desde la última } \\
\text { visita al dentista }\end{array}$} & Menos de 6 meses & 26 & 34,7 & 12 & 48,0 & 8 & 32,0 & 6 & 24,0 \\
\hline & $\begin{array}{l}\text { Entre } 6 \text { meses y un } \\
\text { año }\end{array}$ & 7 & 9,3 & 2 & 8,0 & 2 & 8,0 & 3 & 12,0 \\
\hline & Más de un año & 22 & 29,3 & 6 & 24,0 & 3 & 12,0 & 13 & 52,0 \\
\hline & Nunca & 20 & 26,7 & 5 & 20,0 & 12 & 48,0 & 3 & 12,0 \\
\hline \multirow{5}{*}{$\begin{array}{l}\text { Motivo de la última } \\
\text { consulta }\end{array}$} & Revisión rutinaria & 18 & 24,0 & 7 & 28,0 & 4 & 16,0 & 7 & 28,0 \\
\hline & Ortodoncia & 6 & 8,0 & 2 & 8,0 & 4 & 16,0 & - & - \\
\hline & Caries, dolor & 14 & 18,7 & 3 & 12,0 & 5 & 20,0 & 6 & 24,0 \\
\hline & Otros motivos & 19 & 25,3 & 10 & 40,0 & - & - & 9 & 36,0 \\
\hline & Nunca & 18 & 24,0 & 3 & 12,0 & 12 & 48,0 & 3 & 12,0 \\
\hline \multirow{4}{*}{$\begin{array}{l}\text { Percepción de } \\
\text { la visita al dentista }\end{array}$} & Agradable & 36 & 48,0 & 11 & 44,0 & 12 & 48,0 & 13 & 52,0 \\
\hline & Desagradable & 8 & 10,7 & 2 & 8,0 & - & - & 6 & 24,0 \\
\hline & Indiferente & 2 & 2,7 & - & - & - & - & 2 & 8,0 \\
\hline & $\begin{array}{l}\text { No sabe / } \\
\text { No contesta }\end{array}$ & 29 & 38,7 & 12 & 48,0 & 13 & 52,0 & 4 & 16,0 \\
\hline
\end{tabular}


(36\%) para la edad de 15 años, seguido de Nunca ha visitado al dentista (48\%) a la edad de 12 años y revisión rutinaria (28\%) a la edad de 15 ańos.

La visita al dentista produjo una sensación agradable en el $48 \%$ de los encuestados de 12 años y aumenta hasta el $52 \%$ a los de 15 ańos. Por el contrario la sensación de indiferencia aumenta con la edad al pasar del $0 \%$ al $8 \%$ y un aumento de la sensación desagradable del $8 \%$ a los seis años al $24 \%$ a la edad de 15 años. Ver tabla 1.
El porcentaje de encuestados que nunca habían realizado enjuagues de flúor en el colegio fue aumentando según edades de 6, 12 y 15 ańos en $44 \%, 76 \%$ y $96 \%$ respectivamente. El resto de la muestra lo han realizado al menos durante un año en un $16 \%, 8 \%$ y $4 \%$.

El cepillado diario más de una vez al día es realizado aproximadamente por el $46,7 \%$ de los encuestados y una vez al día por el $21,3 \%$ resultando de la suma de ambos que el cepillado diario lo realizan cerca del $68 \%$. Un $32 \%$ a los 12 años no se cepilla nunca.

Tabla 2. Factores protectores o de riesgo de caries dental

\begin{tabular}{|c|c|c|c|c|c|c|c|c|c|}
\hline \multirow{2}{*}{\multicolumn{2}{|c|}{$\begin{array}{l}\text { Factores protectores o } \\
\text { de riesgo de caries dental }\end{array}$}} & \multicolumn{2}{|c|}{ Total } & \multicolumn{2}{|c|}{6 años } & \multicolumn{2}{|c|}{12 años } & \multicolumn{2}{|c|}{15 años } \\
\hline & & $\mathrm{n}$ & $\%$ & $\mathrm{n}$ & $\%$ & $\mathrm{n}$ & $\%$ & $\mathrm{n}$ & $\%$ \\
\hline \multirow{3}{*}{$\begin{array}{l}\text { Realización } \\
\text { de } \\
\text { enjuagues } \\
\text { de Flúor en } \\
\text { el colegio }\end{array}$} & No nunca & 54 & 72,0 & 11 & 44,0 & 19 & 76,0 & 24 & 96,0 \\
\hline & $\begin{array}{l}\text { Si solo } \\
\text { durante un } \\
\text { año }\end{array}$ & 7 & 9,3 & 4 & 16,0 & 2 & 8,0 & 1 & 4,0 \\
\hline & $\begin{array}{l}\text { Sí, dos o más } \\
\text { años }\end{array}$ & 14 & 18,7 & 10 & 40,0 & 4 & 16,0 & -- & -- \\
\hline \multirow{5}{*}{$\begin{array}{l}\text { Número de } \\
\text { cepillados }\end{array}$} & $\begin{array}{l}\text { Más de una } \\
\text { vez al día }\end{array}$ & 35 & 46,7 & 12 & 48,0 & 4 & 16,0 & 19 & 76,0 \\
\hline & $\begin{array}{l}\text { Una vez al } \\
\text { día }\end{array}$ & 16 & 21,3 & 10 & 40,0 & 2 & 8,0 & 4 & 16,0 \\
\hline & $\begin{array}{l}\text { Menos de } \\
\text { una vez al día }\end{array}$ & 1 & 1,3 & 3 & 12,0 & 1 & 4,0 & -- & -- \\
\hline & $\begin{array}{l}\text { De vez en } \\
\text { cuando }\end{array}$ & 15 & 20,0 & -- & -- & 10 & 40,0 & 2 & 8,0 \\
\hline & $\begin{array}{l}\text { Nunca/casi } \\
\text { nunca }\end{array}$ & 8 & 10,7 & & -- & 8 & 32,0 & -- & -- \\
\hline \multirow{4}{*}{$\begin{array}{l}\text { Ingesta de } \\
\text { alimentos } \\
\text { azucarados } \\
\text { entre } \\
\text { comidas }\end{array}$} & $\begin{array}{l}\text { Todos los } \\
\text { días }\end{array}$ & 16 & 21,3 & 8 & 32,0 & 2 & 8,0 & 6 & 24,0 \\
\hline & $\begin{array}{l}1 \text { o } 2 \text { días por } \\
\text { semana }\end{array}$ & 25 & 33,3 & 7 & 28,0 & 14 & 56,0 & 4 & 16,0 \\
\hline & $\begin{array}{l}\text { Con menor } \\
\text { frecuencia }\end{array}$ & 15 & 20,0 & 6 & 24,0 & -- & -- & 9 & 36,0 \\
\hline & $\begin{array}{l}\text { Nunca/casi } \\
\text { nunca }\end{array}$ & 19 & 25,3 & 4 & 16,0 & 9 & 36,0 & 6 & 24,0 \\
\hline
\end{tabular}

Tabla 3. Factores protectores o de riesgo de la caries dental

\begin{tabular}{|c|c|c|c|c|c|c|c|c|c|}
\hline \multirow{2}{*}{\multicolumn{2}{|c|}{$\begin{array}{l}\text { Factores protectores o de } \\
\text { riesgo de la Caries dental }\end{array}$}} & \multicolumn{2}{|c|}{ Total } & \multicolumn{2}{|c|}{6 años } & \multicolumn{2}{|c|}{12 años } & \multicolumn{2}{|c|}{15 años } \\
\hline & & $\mathrm{n}$ & $\%$ & $\mathrm{n}$ & $\%$ & $\mathrm{n}$ & $\%$ & $\mathrm{n}$ & $\%$ \\
\hline \multirow{4}{*}{$\begin{array}{l}\text { Conocimiento } \\
\text { de la acción } \\
\text { del flúor sobre } \\
\text { el diente }\end{array}$} & Protege & 28 & 37,3 & 10 & 40,0 & 6 & 24,0 & 12 & 48,0 \\
\hline & Refresca & 7 & 9,3 & 3 & 12,0 & 4 & 16,0 & -- & -- \\
\hline & Blanquea & 20 & 26,7 & 7 & 28,0 & 7 & 28,0 & 6 & 24,0 \\
\hline & $\begin{array}{l}\text { No sabe/No } \\
\text { contesta }\end{array}$ & 20 & 26,7 & 5 & 20,0 & 8 & 32,0 & 7 & 28,0 \\
\hline \multirow{3}{*}{$\begin{array}{l}\text { Conocimiento } \\
\text { sobre si es } \\
\text { beneficioso } \\
\text { ingerir } \\
\text { alimentos } \\
\text { entre comidas }\end{array}$} & Sí & 23 & 30,7 & 6 & 24,0 & 7 & 28,0 & 10 & 40,0 \\
\hline & No & 30 & 40,0 & 12 & 48,0 & 11 & 44,0 & 7 & 28,0 \\
\hline & $\begin{array}{l}\text { No sabe/no } \\
\text { contesta }\end{array}$ & 22 & 29,3 & 7 & 28,0 & 7 & 28,0 & 8 & 32,0 \\
\hline
\end{tabular}

A los 15 años el porcentaje de niños que decía ingerir alimentos azucarados todos los días entre comidas disminuye de $32 \%$ a la edad de seis años a $8 \%$ y aumenta a $24 \%$ a la edad de 12. La ingesta de alimentos azucarados altamente cariogénicos una o dos veces a la semana la realizaron el $56 \%$ de los encuestados de 12 años y el $16 \%$ de los de 15 ańos. Ver tabla 2.

La acción protectora del flúor es conocida por $24 \%$ a los 12 ańos y aumenta hasta el $48 \%$ a los 15 años. Un $28 \%$ a los 12 ańos cree erróneamente que blanquea los dientes, incluso $16 \%$ cree que refresca.

Un 30,7\% conocen que la ingestión de alimentos entre comidas no es beneficiosa para su salud oral. El 29,3 \% no sabe o no contesta. Ver tabla 3.

La mayor parte de los encuestados, un porcentaje mayor al $80 \%$, como en el caso de golosinas y caramelos, conoce la capacidad cariogénica de los distintos grupos de alimentos, excepto en el grupo de huevos y leche (68\%), verdura y ensalada $(70,7 \%)$ donde existió un mayor desconocimiento, cuando se abordó sobre la creencia de cuál de los alimentos citados son perjudiciales para sus dientes. Ver Fig. 1.

\section{Discusión}

En cuanto a los hábitos de higiene, el cepillado dental diario se incrementa ligeramente al pasar de un $8 \%$ en la edad de 12 años a un $16 \%$ de los escolares de 15. El porcentaje de los que se cepillan más de una vez al día variaron de $16 \%$ a la edad de los 12 años a $76 \%$ a la edad de 15 años. El porcentaje de escolares que manifiesta no cepillarse nunca se da en la edad de 12 años con un $32 \%$. Se necesita establecer en el futuro una evaluación de los hábitos de higiene de cepillado dental.

Los datos encontrados en porcentaje son muy bajos comparados con los escolares de la comunidad Valenciana en un estudio del $2004^{11}$ en el que se encontró porcentajes en cuanto a los hábitos de higiene, el cepillado dental diario se incrementa ligeramente al pasar de un $73,9 \%$ en 1998 a un 78,7 $\%$ de los escolares de 12 ańos en 2004 (52,2\% más de una vez). El porcentaje de escolares que manifiesta no cepillarse nunca se ha reducido de un $20 \%$ a un $12 \%$ al pasar de los 6 ańos a los 15 años. Los resultados son distintos a los obtenidos en otras comunidades autó- 
nomas de España donde se ha reducido de 3,0 \% a 2,4\%. En el presente estudio los porcentajes de niños que se cepillaban más de una vez al día fue de $46,7 \%$ frente a otros estudios como el estudio internacional de la OMS ${ }^{12}$ llevado a cabo en colaboración con el Center of Health Administration Studies de la Universidad de Chicago que recogía encuestas realizadas entre 1988 y 1992 muestran porcentajes de niños que se cepillaban más de una vez al día que oscilan entre el $46 \%$ y $73 \%$. En el estudio de Kusela y cols ${ }^{13}$ de 1994 que abarcaba más de 20 países europeos, los más de una vez al día oscilaban entre el $83 \%$ de Suecia y Dinamarca y el $34 \%$ de Rusia y Lituania.

Uno de los pilares básicos en los que se basa la acción preventiva de la caries en la Comunidad Valenciana es el programa de enjuagues fluorados en las escuelas. En 1998 un 52,3 \% de los escolares había realizado enjuagues durante dos o más cursos. En 2004 este porcentaje se había elevado hasta el 56,7 \%. Otro apunte favorable destaca por el hecho de que el porcentaje de escolares que nunca ha realizado enjuagues ha descendido en este periodo del 23,3\% al 17,5 \%. En el Perú el Programa Nacional de salud Bucal dejó de funcionar el programa de enjuagues fluorados en las escuelas.

El conocimiento de la acción preventiva del flúor en el presente estudio es conocida por $24 \%$ a los 12 ańos y aumenta hasta el $48 \%$ a los 15 años frente a los presentados en el estudio de la comunidad Valenciana que se mantiene desde el año 1998 donde el $83 \%$ de los escolares de entre 12 y 16 años sabía que el flúor tiene una acción beneficiosa para los dientes. Hay un $16 \%$ que no sabe la acción beneficiosa del flúor. porcentajes de niños que se cepillaban

La frecuencia de escolares que nunca ha visitado al dentista a los doce años es de $48 \%$, cifra muy alta en comparación con escolares de la comunidad Valenciana en la que cada vez es mayor el porcentaje de nińos de entre 12 y 16 años que había ido al dentista alcanza valores entre el 73-92\%.

En cuanto a la capacidad cariogénica de algunos alimentos se clasifican como cariogénicos a huevos y leche un $68,0 \%$, verduras y ensaladas $70,7 \%$.

\section{Conclusión}

1. En general, los hábitos higiénicos de los escolares de la IE. 5130 son insatisfactorios debido principalmente a la poca frecuencia de cepillados diarios $46,7 \%$, a la no realización durante periodos más prolongados de enjuagues de flúor en el colegio $(72,0 \%)$ y en las pocas visitas al dentista $(34,7 \%$ en menos de 6 meses; 29,3\% más de un ańo).

2. Los conocimientos de los escolares acerca de la acción del flúor sobre el diente son inadecuados en las edades de 6, 12 y 15 ańos en un $60 \%, 76 \%$ y $52 \%$.

3. Es imperiosa la necesidad de brindar programa preventivo promocional, intervención y asistencial a la población escolar de la IE. 5130.

\section{Referencias bibliográficas}

1. Rose G. Sick individuals and sick populations. Int J Epidemiol, 1985; 14:32-38.

2. Ll, serra-Majem y E. Cuenca. Prevención e Investigación en Odontología y Salud pública. En: Métodos de investigación en odontología. Ed. Masson,2000;1:1-12.

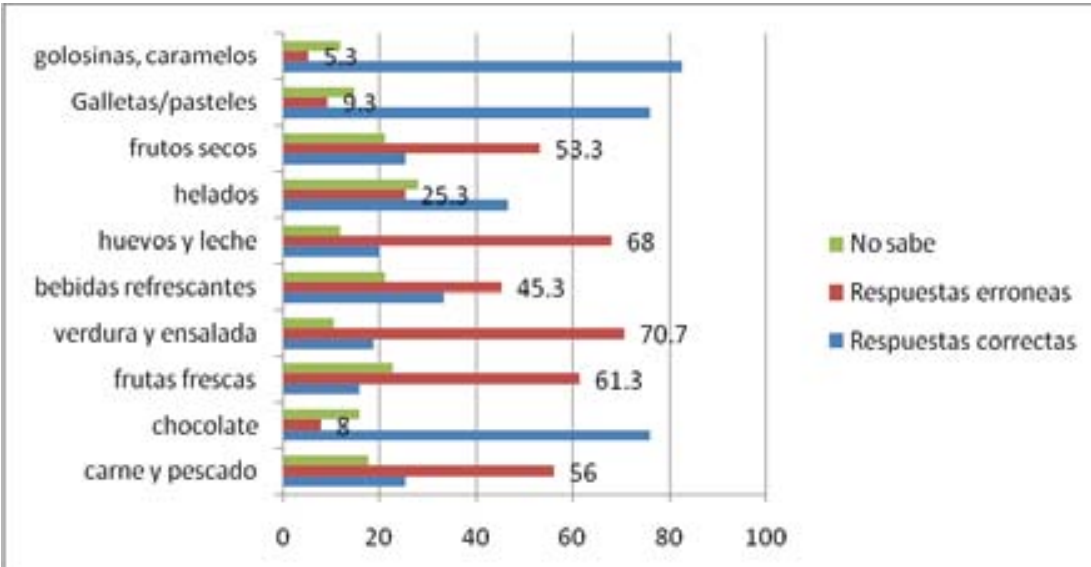

Figura 1. Porcentajes globales de respuesta acerca del grado de cariogenicidad de los distintos grupos de alimentos (incluyen 6, 12 y 15 ańos).
3. Cuenca Sala E. caries: fundamentos actuales de su prevención y control. En: Cuenca E, Manau C, Serra L eds. Odontología preventiva y comunitaria: principios, métodos $\mathrm{y}$ aplicaciones. Barcelona: Masson, 1999; 15-29.

4. Cuenca Sala E. odontología Comunitaria. Evolución histórica, principios y aplicaciones. En: Cuenca E, Manau C, Serra L,eds. Odontología preventiva y comunitaria: principios, métodos y aplicaciones. Barcelona: Masson, 1999, 249-253.

5. Cuenca Sala E. Odontología preventiva y comunitaria- Principios, métodos y aplicaciones. Elsevier Masson. 2005;1:1-17

6. World health Organization. Otawa Charter for Health Promotion. Géneve WHO.1986.

7. Schou K, Locker D. Priciples of oral health promotion. En: Pine C, editor. Community Oral Health. Oxford: Wright;1997.pg.177-86

8. Burt B. Promotion of oral health. En: Burt Beklund S, editors. Dentistry, dental practice and the community. $4^{\text {th }}$ ed. Philadelphia: saunders, 1992.p.241 -52.

9. BrathallD, Hänsel-Petersson G, Sanberg $\mathrm{H}$. Reasons for the caries decline: What do the experts believe? Eur J Oral Sci. 1996;104:116-22.

10. Organización Mundial de la Salud. Encuestas de salud bucodental: métodos básicos de la 4ta ed. Ginebra. Organización Mundial de la Salud. 1997.

11. Almerich-Silla, José Manuel y Montiel-Company, José María. Encuesta sobre hábitos higiénicos orales en la población adolescente de la comunidad Valenciana (2004). RCOE 2006;11(2):195-201.

12. Chen N, Andersen RM, Barmes DE, Leclerq MH, Lyttle CS. Comparing Oral Health Care Systems: a second international collaborative study World Health Organization: Geneva. 1997.

13. Kusela S, Honkala S, Kannas L, Tynjälä J, Wold B. Oral Hygiene Habits of 11-year-old school children in 22 countries and Canada in 1993-94. J Dent Res 1997;76:1602-9. 